\title{
Mining-Induced Seismicity Associated with Self-Similar Propagation of Sliding Zones
}

\author{
A.V. Dyskin School of Civil and Resource Engineering, The University of Western Australia
}

\begin{abstract}
Mining operations near faults can induce sliding zones which grow further driven by the excess of the shear stress over friction at the loci of initiation. To model interaction between the sliding zones we assume, guided by the GuttenbergRichter law, that the distribution of their sizes is self-similar and the self-similarity is maintained in the process of their growth. We show that the latter is only possible if the sliding zones are coplanar and that the exponent and the prefactor of the distribution function are uniquely determined. The addition of a new sliding zone does not change the distribution but rather increases the upper cut-off. This happens either by instantaneous growth of the added zone to the maximum size producing the strongest microseismic event or by initiating a cascade of intermediate crack growth producing a series of smaller events. The found energy distribution permits risk assessment based on the determination of the probability of hazardous events. The model parameters can be determined by observing statistical moments of the energy distribution combined with the determination of fault deformation from its influence on the approaching excavations by displacement monitoring.
\end{abstract}

\section{INTRODUCTION}

Mining-induced fault sliding and the resulting seismic event pose a considerable hazard to both the excavations and the environment (see for instance Gibowicz and Kijko, 1994 and literature therein). Thus the problem of predicting high magnitude events is very important and received considerable attention in the literature. The major approach to theoretical prediction of dangerous fault sliding is to determine whether the conditions of sliding are satisfied and if yes, then within which area (e.g., Galybin and Odintsev, 1993).

In principle, this approach allows complete determination of the magnitude of the resulting seismic event and, subsequently, the amount of damage to the excavation and the environment provided that the key parameters of the rockmass and the fault are known. The key parameters in this case are the original stress state, the type and parameters of the rockmass constitutive behaviour and the strength properties of the fault (for instance the parameters of the friction law). It should be emphasised that while the determination of these parameters is hampered by the scale effect, the determination of the stress state and the rockmass deformability can be assisted by modelling the stress and displacement produced by the excavation and than back analysing the results of stress and displacement monitoring.

The most difficult is however the determination of the fault strength properties, since the parameters determined by retrospective analysis of the post failure situation (postmortem examination) do not have to be relevant to a next sliding which will happen in conditions changed by the previous fault sliding. In this case the main tool for prediction of the sliding or, at least the risk assessment, is microseismic monitoring. The major challenge in microseismic monitoring is the development of interpretation methods which, in order to be realistic, have to be based on the understanding the mechanics of catastrophic fault sliding.

In many cases the mechanical behaviour of the fault is controlled by the rockmass structure encompassing a number of scales. Then the assumption of self-similarity in distributions of microstructural elements becomes a major simplifying factor in an otherwise usually intractable problem. There is a strong evidence of self-similar properties of both rocks and the Earth's crust (e.g., Sadovskiy, 1983; Scholz and Aviles, 1986; Scholz, 1990; Redner, 1990; Olding, 1992; Barton and Zoback, 1992; Turcotte, 1993; Gillespie et al., 1993; Yamamoto et al., 1993; Dubois, 1998).

The appearance of self-similar structures is usually attributed to the critical state of the material (e.g., Bak and Tang, 1989; Chopard and Droz, 1998), however, the particular mechanism of formation of self-similar distributions, particularly distributions of cracks and fractures, is poorly understood. The most popular approach is to consider the fractures as clusters of connected defects (e.g., Sahimi and Goddard, 1986; Nishiuma et al., 1996; Chakrabati and Benguigui, 1997; Mishnaevsky, 1998) which, near the critical state (i.e. at the percolation threshold), have self-similar distributions. It should however be noted that only in the $2 \mathrm{D}$ picture these structures actually break the material. In the real 3D world the formation of such structures does not affect the connectedness of the body.

Dyskin $(2001,2002)$ proposed a mechanism of developing isotropic self-similar distributions of disk-like cracks, based on crack interaction and leading to a self-similar distribution of crack sizes, with the distribution function proportional to the inverse fourth power of the crack radius. Essential in this model is the stable growth of the cracks, which is provided by a special type of loading, viz by a couple of concentrated forces applied at the centre of every disk-like crack. This paper develops this approach and applies it to the parallel self-similar fault systems.

It is important to emphasise that the approaches based on self-similarity, in particular the concept of fractals are quite crude, since they deal mainly with the scaling exponent that determine the transition form one scale to another. This, despite of the crudeness, is an advantage for rock mechanics problems since it allows analysis in situations with restricted information and provides a basis for scaling the laboratory data to the scales relevant to the mining field situations. 


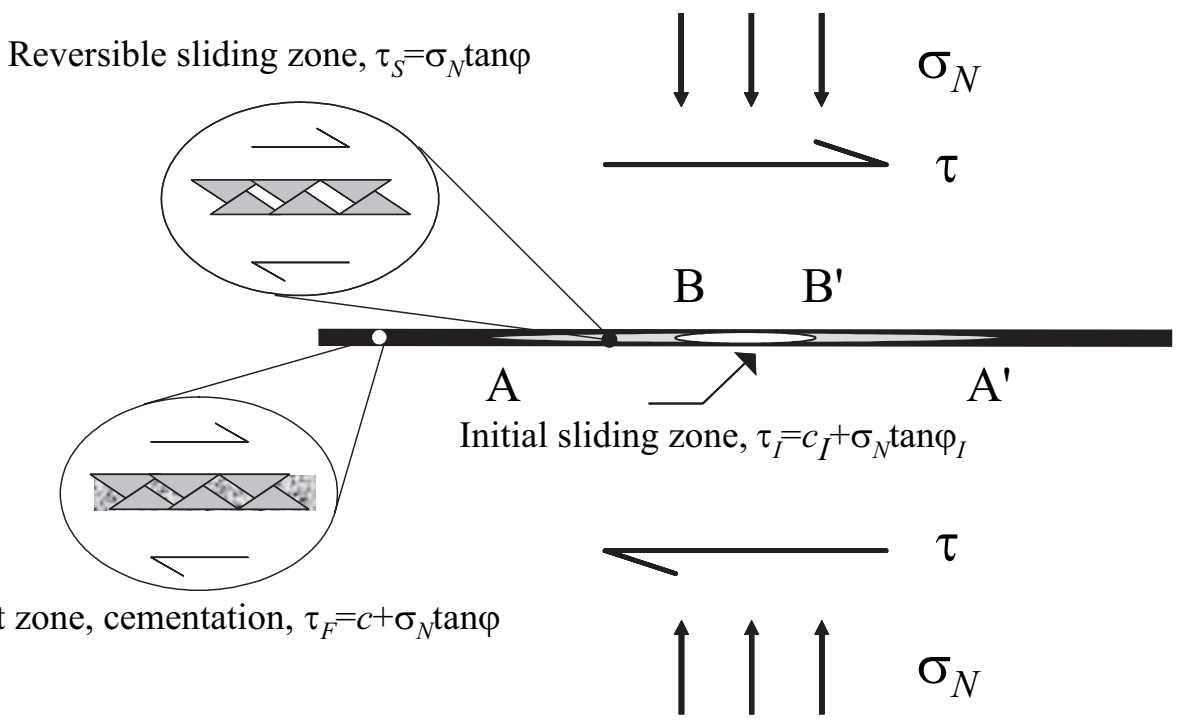

FIG. 1 A schematic 2D representation of a fault with a sliding zone AA' developed from an initial sliding zone BB'

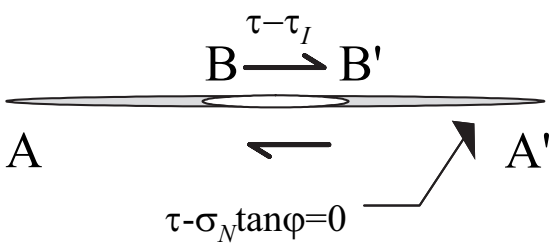

(a)

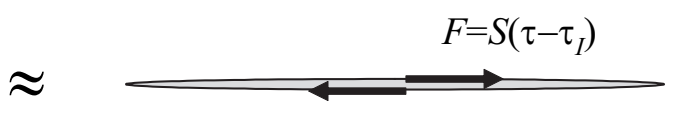

(b)

FIG. 2 A model of the sliding zone: (a) a representation of the sliding zone as a shear crack; (b) modelling the sliding zone as a shear crack loaded by a pair of concentrated forces

\section{MODEL OF A SLIDING ZONE}

Consider a fault (or a system of parallel faults) and suppose the fault is in a meta-stable condition meaning that after a previous sliding there was sufficient time for a cementation process to restore the cohesion. We will express this condition in terms of the large-scale in situ shear, $\tau$, and normal, $\sigma_{N^{\prime}}$ stress components, acting on the fault plane assuming that they satisfy the following conditions

$$
\tau<c+\sigma_{N} \tan \varphi, \quad \tau=\sigma_{N} \tan \varphi
$$

where $\varphi$ is the friction angle and $c$ is the cohesion created for instance by fault cementation.

Mining operations undertaken in a vicinity of the fault can generate local sliding zones, which, as yet, do not affect the fault stability. These zones appear at weak places due to change in the stress state caused by advancing excavations or due to local friction deterioration caused for instance by cyclic loading due to blasting. The local sliding zones can also be formed in the situation when the fault is locally hit by a tensile wave with a magnitude exceeding the normal compressive stress (this mechanism was considered by Dyskin et al., 1998). We will call these zones the initial sliding zones. The initial sliding zone (denoted by BB' in Figure 1) is characterised by the sliding resistance, $\tau_{\mathrm{I}}$, which is considerably lower than the large-scale friction on the fault:

$$
\tau_{I}=c_{I}+\sigma_{N} \tan \varphi_{I}<\sigma_{N} \tan \varphi
$$

The initial sliding zone will propagate to a sliding zone of a certain length (denoted by $\mathrm{AA}^{\prime}$ in Figure 1). We will assume that the cementation in this zone is broken and the resistance to sliding is only provided by dilation on asperities in accordance to a conventional model (Patton, 1966).

In the case when the sliding zone is alone (interacting self-similarly distributed sliding zones will be considered in the following sections) its development can be modelled by a shear crack laded at segment $\mathrm{BB}^{\prime}$ by shear tractions $\tau-\tau_{\mathrm{I}}$, Figure $2 \mathrm{a}$. Since we are going to consider potentially extensive development of the sliding zones which are much greater than the size of the initial zone we will, as a further simplification, model the sliding zone as a crack sheared by a pair of concentrated forces of average magnitude $F=S\left(\tau-\tau_{\mathrm{I}}\right)$, where $S$ is the area of the initial sliding zone, Figure $2 b$.

The growth of such sliding zones is controlled by the fault microstructure, in particular the fault roughness and the strength of the gouge. Macroscopically, these factors will be represented by Mode II fracture toughness, $K_{\text {IIc }}$ assumed to be scale-independent. Then the crack propagation type criterion can be used as a criterion of the sliding zone growth:

$$
K_{I I}=K_{I I c}
$$

For the crack shown in Figure 2b, the Mode II stress intensity factor $K_{I I}$ has the following form (e.g., Tada et al., 1985)

$$
K_{I I}=\frac{F}{\sqrt{\pi l / 2}}
$$

where $l$ is the crack length.

As evident from equations (3) and (4), the sliding zone grows in a stable manner with the radius increasing as $F^{2}$. This imposes bonds on the sizes of the sliding zones meaning that the growth of separate sliding zones by themselves cannot 
cause catastrophic sliding. The mechanism of large seismic events should therefore be sought in the effect of interaction between the sliding zones. This mechanism is considered in the following section.

\section{EMERGENCE OF SELF-SIMILARITY IN DISTRIBUTIONS OF INTERACTING CRACKS}

Interaction between sliding zones literally means that each zone propagates driven by a superposition of the external stress and stress disturbances, generally non-uniform, generated by all other zones. When the number of sliding zones is large the modelling of propagation of interacting sliding zones becomes quite complicated.

A considerable simplification can be achieved by employing the notion of self-similarity in the distribution of the sliding zones, which follows from the GuttenbergRichter law universally observed in the records of both natural and mining-induced seismic events (e.g., Gibowicz and Kijko, 1994). Then the fact that self-similarity implies that the scaling is described by the power law, is the major simplifying factor in addressing the problem of interaction as will be shown in the following section. More importantly, in some cases, including the case of sliding zones considered, the interaction itself can produce self-similar distributions as will be demonstrated below (the consideration below will use the method developed by Dyskin $(2001,2002)$ for Mode I cracks in 3D).

Consider a material with cracks growing in a stable manner, for instance driven by pairs of equivalent concentrated forces applied to the centres of the crack faces. Suppose that the cracks are located randomly. Then, even if all cracks were initially of the same size and were loaded by exactly the same forces, the interaction will make them grow differently such that a certain size distribution of cracks will emerge. One can then assume that the difference in the crack sizes will only increase with their growth. Thus the interaction of such cracks can be modelled in the asymptotics of large distribution of sizes (Salganik, 1973) assuming that: (i) cracks of close sizes do not interact directly and; (ii) the interacting cracks are very different in size. Then each crack can be considered in an equivalent medium with effective characteristics determined by all cracks of smaller sizes.

We will start the consideration with a somewhat artificial case of isotropic distribution of sliding zones (shear cracks), meaning that the faults subject to sliding are randomly oriented. We will further assume that the asperity-resisted sliding can be reversible such that small deviations of the shear stresses from the friction stress can cause both increase and reduction in the zone sliding. This assumption is essential for the proposed use of the effective characteristic theory since the basis of it - the consideration of a crack in an effective medium determined by smaller cracks - presumes that the effective medium is elastic at least in a vicinity of the equilibrium point, including the assumption that loading and unloading in that vicinity follow the same path. Modelling of this case will provide the concept which will then be extended to the realistic case of parallel faults.

For isotropic distribution of sliding zones the average stress intensity factor $\left\langle K_{I I}>\right.$ is given by (see Dyskin, 2002 for details)

$$
\frac{\left\langle K_{I I}\right\rangle}{K_{I I}{ }^{0}} \sim \frac{E_{0}}{E}
$$

where $E$ is the effective Young's modulus, $E_{0}$, is the Young's modulus of the material, $K_{I I}{ }^{0}$ is the stress intensity factor for the shear crack without interaction. Then the criterion of crack propagation (3), (4) assumes the form

$$
K_{I I c}=\frac{F}{\sqrt{\pi l / 2}} \frac{E_{0}}{E}
$$

Suppose that at a certain value of the load, $F$, the cracks have the distribution function $f(l), l_{0} \leq l \leq l_{\max }$. Here $l_{0}$ is the length the crack would have without the interaction, $l_{\max }$ is the maximum length attained under the given value $F$. It will be assumed that this crack distribution can approximately be considered as the wide distribution of sizes.

In accordance with this method, the effective moduli for the material with cracks up to the given length should be calculated. Consider cracks with lengths between 1 and $1+\mathrm{dl}$. These cracks shall be considered as non-interacting and placed in an effective medium determined by cracks of lengths less than 1. Let the Young's modulus and Poisson's ratio of this medium be E(l) and v(l). Then, following Salganik's (1973) method, the effective characteristics for the medium with the new cracks of lengths between 1 and $1+\mathrm{dl}$ are obtained as the effective characteristics of a medium with moduli $\mathrm{E}(\mathrm{l})$ and $v(1)$ with non-interacting cracks of concentration:

$$
d \Omega(l)=N l^{2} f(l) d l
$$

where $N$ is the number of cracks per unit area.

For the case of pure shear cracks (no opening or closure, i.e. normal displacement is continuous through the crack) the effective moduli in the approximation of low concentrations $(\Omega<<1)$ have the following form (e.g. Krajcinovic, 1996):

$$
E=E_{0}\left(1-\frac{\pi}{16} \Omega\right), \quad v=v_{0}+\left(1-v_{0}\right) \frac{\pi}{16} \Omega
$$

Here, for the sake of simplicity, only the plane stress conditions are considered. The plane strain situation can be found by formal replacement $E \rightarrow E\left(1-v^{2}\right)^{-1}, v \rightarrow v(1-v)^{-1}$. According to the differential self-consistent method, at each step that we apply formula (8) the moduli at the previous step play the role of $E_{0}$ and $v_{0}$ in the approximation of low concentrations, such that

$$
\begin{aligned}
& E(\Omega+d \Omega)=E(\Omega)\left(1-\frac{\pi}{16} d \Omega\right) \\
& v(\Omega+d \Omega)=v(\Omega)+(1-v(\Omega)) \frac{\pi}{16} d \Omega
\end{aligned}
$$

We now obtain from the first equation that

$$
\frac{d E}{d l}=-\frac{\pi}{16} E\left(N l^{2} f(l)\right)
$$

Expressing $E$ from (6) differentiating it with respect to $l$ and substituting into (9) one obtains

$$
f(l)=\frac{8}{\pi N l^{3}}
$$

The distribution function should satisfy the usual normalisation condition

$$
\int_{l_{0}}^{l_{\max }} f(l) d l=1
$$

From here one obtains

$$
\begin{aligned}
& l_{\text {max }} / l_{0}=\left[1-\left(F / F_{\max }\right)^{4}\right]^{-1 / 2} \\
& F_{\text {max }}=K_{\text {IIc }}\left(\frac{N}{4 \pi}\right)^{1 / 4}, \quad l_{0}=\frac{2}{\pi}\left(\frac{F}{K_{I I c}}\right)^{2}
\end{aligned}
$$


where $N$ is the number of cracks per unit area, $F_{\max }$ is the force magnitude at which the maximum crack length becomes infinite, which can be interpreted as the material failure. The lower boundary, $l_{0}$ corresponds to the crack growth "not assisted" by the interaction.

The power law (10) together with $l_{\max } / l_{0} \rightarrow \infty$ can be interpreted as an emergence of a self-similar distribution in the crack arrangement considered. In more complex cases of crack arrangement it is technically more difficult to trace the emergence of self-similar distributions. What can be done instead is to determine whether the self-similar distributions are stable with respect to the described type of crack growth. This, necessary condition of the self-similar distributions will be analysed in the following sections.

\section{MECHANICS OF MATERIALS WITH SELF-SIMILAR CRACK SETS}

Let the crack distribution be self-similar such that there is no characteristic size in the microstructure. According to Dyskin (2004) a material with such crack distribution should be modelled simultaneously at all scales by a continuous set of continua (the $H$-continua) with the volume element sizes, $H$, assuming all values. In this case, all continuum quantities should be also functions of scale, $H$. Then all characteristics of the continua become the power functions of $H$. Furthermore, it was proven that all tensorial properties should scale isotropically, i.e. all tensorial components should scale with the same exponent. In particular, the tensors of elastic moduli, $\mathbf{C}$, and compliances, $\mathbf{A}$, in a Cartesian co-ordinate frame $x_{1^{\prime}}$ $x_{2}, x_{3}$ must scale for any crack orientations and any material anisotropy as

$$
\begin{aligned}
& C_{i j k l}(H)=c_{i j k l} H^{\alpha}, \quad A_{i j k l}(H)=a_{i j k l} H^{\beta} \\
& i, j, k, l=1,2,3, \quad \alpha=-\beta
\end{aligned}
$$

for all non-zero components of the prefactors.

The prefactors and exponents can be determined from the following system of equations, if the expression for the scaling for the contribution of cracks to the compliances or moduli, $\Delta A_{i j k l}=\Delta A_{i j k l} H^{\gamma}$ or $\Delta C_{i j k l}=\Delta C_{i j k l} H^{\gamma}$ are known for the case of non-interacting cracks (sic!). Then using the differential selfconsistent method and taking into account that according to the dimension analysis $\gamma=\beta-1$ (or $\gamma=\alpha-1$ ) one obtains the following system of scaling equations (see details in Dyskin, 2004)

$$
\beta a_{i j k l}=\Delta a_{i j k l} \quad \text { or } \quad \alpha c_{i j k l}=\Delta c_{i j k l}
$$

Each of the above systems is generally a system of 21 equations for 22 unknowns, $a_{i j k l}$ and $\beta$. Since the prefactors for both compliances and the increments have the same units, one of the compliance prefactors can be chosen arbitrarily, while the other prefactors and the exponent can be found from equation (14).

Furthermore, in line with equation (5), the average SIFs, in particular the Mode II SIF, scale as

$$
<K_{I I}(H)>\sim H^{\alpha}
$$

For a special case of self similar crack distributions $f(l)=\omega l^{-3}$, to which distribution (10) belongs (with the concentration factor $\omega=8 / \pi N)$, it is shown by Dyskin (2002) that the assumptions of the wide distribution of sizes are satisfied and that the differential self-consistent method can be used to determine $\Delta c_{i j k l}$.

In the case of randomly oriented mode II cracks the second system of scaling equations (14) can be obtained from equation (8) by considering only the crack contribution to the moduli and then by formal replacement of $\Omega$ with $\omega$ :

$$
\alpha E=-E \frac{\pi}{16} \omega, \quad 0=(1-v) \frac{\pi}{16} \omega
$$

The solution of equation (16) reads

$$
E=e H^{\alpha}, \quad \alpha=-\frac{\pi}{16} \omega, \quad \nu=1
$$

where $e$ is a normalising prefactor. It is seen that both Young's modulus and Poisson's ratio scale according to power laws. The exponent for the Young's modulus is proportional to the concentration factor $\omega$, while the exponent for the Poisson's ratio is zero because the Poisson's ratio is bounded. It is interesting that the value for the plane stress Poisson's ratio is found to be 1 , which after the replacement $v \rightarrow v(1-v)^{-1}$ gives that the value of Poisson's ratio is 0.5 , suggesting that materials with self-similar distributions of isotropically oriented pure Mode II cracks are incompressible. This is because formally, for finite $\omega$, the total crack concentration is infinite such that the properties of materials with selfsimilar crack distributions are fully controlled by the crack behaviour. In this case the incompressibility is a result of the absence of any normal relative displacements of the faces of Mode II cracks.

Consider now a case of parallel Mode II cracks which could model sliding over a distributed set of parallel faults. Suppose the cracks are oriented perpendicular to the $x_{2}$ axis and distributed self-similarly with the distribution function $f(l)=\omega l^{-3}$. For this case the effective compliances can be found from the general solution by Vavakin and Salganik (1978) in which the contribution of the crack in the normal strain in the direction perpendicular to the cracks (the $x_{2}$ axis) should be set to zero:

$$
\left\{\begin{aligned}
A_{11} & =A_{11}{ }^{0} \\
A_{22} & =A_{22}{ }^{0} \\
A_{66} & =A_{66}{ }^{0} \\
& +\frac{\pi}{4} \Omega \sqrt{{A_{11}}^{0}\left(2{A_{12}}^{0}+{A_{66}}^{0}+2 \sqrt{\left.{A_{11}{ }^{0} A_{22}{ }^{0}}^{2}\right)}\right.} \\
A_{12} & =A_{12}{ }^{0}
\end{aligned}\right.
$$

Here $A_{11}{ }^{0}, A_{22}{ }^{0}, A_{12}{ }^{0}, A_{66}{ }^{0}$ are the compliances of the material, such that the Hook's law has a form:

$$
\begin{aligned}
& \varepsilon_{11}=A_{11}{ }^{0} \sigma_{11}+A_{12}{ }^{0} \sigma_{22} \\
& \varepsilon_{22}=A_{12}{ }^{0} \sigma_{11}+A_{22}{ }^{0} \sigma_{22} \\
& \varepsilon_{12}=1 / 2 A_{66}{ }^{0} \sigma_{12} .
\end{aligned}
$$

The scaling equations (the first system in equation (14)) can be obtained by replacing $\Omega$ with $\omega$ bringing $A_{i i}{ }^{0}$ to the lefthand sides and then replacing $A_{i i}-A_{i i}{ }^{0}$ with $\beta a_{i i}{ }^{0}$ and, finally, replacing $A_{i i}{ }^{0}$ with $a_{i i}$ in the remaining parts. This yields the following scaling equations

$$
\left\{\begin{array}{l}
\beta a_{11}=0 \\
\beta a_{12}=0 \\
\beta a_{22}=0 \\
\beta a_{66}=\frac{\pi}{4} \omega \sqrt{a_{11}\left(2 a_{12}+a_{66}+2 \sqrt{a_{11} a_{22}}\right)}
\end{array}\right.
$$

If $\beta \neq 0$ then the first equation of (20) yields $a_{11}=0$ and then the last equation produces $\beta=0$. This results in the following scaling laws:

$$
A_{i j}=a_{i j} H^{\beta}, \quad \beta=0, \quad a_{11}=0
$$

$a_{66}$ being a normalising constant. 
The result that the compliance $a_{11}$, that relate normal stress and strain components, is zero, is not surprising since it merely reflects the fact that a parallel set of mode II cracks only reduces the shear compliances $\left(a_{66}\right)$. Given that formally the total crack concentration is infinite the other compliances become infinitely small as compared to $a_{66}$. What could not be predicted without solving the scaling equations is the trivial scaling of $a_{66}$. It should be noted that the same situation will take place in the case of two sets of mutually orthogonal Mode II cracks. This trivial scaling will have a profound effect on the stability of this crack distribution with respect to their growth as is explained in the following section.

\section{STABILITY OF DISTRIBUTED SELF-SIMILAR SETS OF GROWING CRACKS}

We are now in a position to check whether the above distributions of Mode II cracks are stable with respect to the mechanism of crack growth described in Section 2. Assuming that each homogenisation scale $H \sim l$ (in the $H$-continuum only cracks of sizes $l>H$ can be seen) and then substituting equation (15) into the expression for the stress intensity factor (4) and then into the criterion of crack growth equation (3) one obtains

$$
K_{I I c}=K_{I I} \sim l^{-\alpha-1 / 2}
$$

From here, since the fracture toughness, $K_{I I c^{\prime}}$ is assumed to be scale independent: $-\alpha-1 / 2=0$. Therefore, if the cracks are to grow keeping the self-similarity the exponent of the moduli scaling cannot be arbitrary, but should satisfy

$$
\alpha=-1 / 2
$$

For isotropic distributions of Mode II cracks this, according to equation (17) corresponds to

$$
\omega=8 / \pi
$$

Thus the concentration factor of the crack distribution remains constant such that the crack growth only affects the lower and upper cutoffs of the distribution (both $l_{\max }$ and $l_{0}$ get increased in accordance with equations (12)).

In the case of parallel Mode II cracks condition (23) cannot be satisfied due to the trivial scaling $(\alpha=\beta=0)$. Therefore, for such crack distributions, the self-similarity cannot be maintained: it will be destroyed by crack growth. Consequently, one cannot expect that the crack growth will be accompanied by self-similar seismic emission which contradicts the Guttenberg-Richter law. This suggests that the case of distributed cracks locations is not realistic and a situation of cracks localised in a narrow band should be considered. This will be accomplished in the following section.

\section{LOCALISED SETS OF PARALLEL CRACKS. STABILITY OF SELF-SIMILAR DISTRIBUTIONS}

The instability of a self-similar distribution of parallel cracks with respect to the crack growth came from the fact that the scaling exponent vanishes. This, in its own turn, is a consequence of the fact that these cracks do not contribute to some compliances that characterise the orthotropic $H$ continua which model the material with one set of parallel cracks. Therefore, in order to find a stable arrangement of parallel cracks, one needs to find a situation when the cracks influence all essential components of compliances. An obvious candidate for this is a localised distribution of parallel cracks, i.e. the distribution in which all cracks are concentrated within a thin layer, Figure 3. (In order to maintain the self-similarity the layer should be infinitesimally thin; in reality its thickness should be much smaller than the lower cutoff of the crack lengths, $l_{0}$.) We will model such a set of coplanar cracks as a Winkler layer with the shear stiffness $k$ defined as $\tau=k \Delta u_{s^{\prime}}$ where $\Delta u_{\mathrm{s}}$ is the shear displacement discontinuity over the layer (difference in displacements at opposite boundaries of the layer) in response to shear load $\tau$.

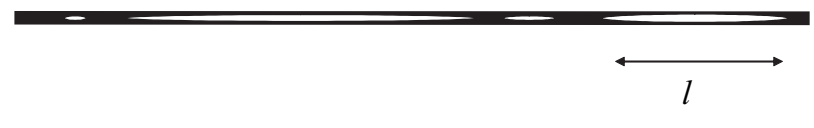

\section{FIG. 3 Coplanar sliding zones localized in the fault}

Under the assumption of self-similar crack distribution, the stiffnesses should scale with the same exponent (since they are components of a diagonal tensor relating the stress vector and displacement discontinuity vector):

$$
k \sim H^{\alpha}
$$

Suppose the cracks are distributed as follows

$$
f(l)=\frac{\lambda}{l^{m}}
$$

Let an $H$-continuum comprise all cracks of the size up to $l \sim H$. Transition to the scale $H+d H$ leads to the addition of new cracks occupying the relative length of the fault (the area per unit length in the direction normal to the drawing on Figure 3) $d \omega=\lambda l^{1-m} d l$. These new cracks increase the average (effective) stress, $\tau_{\text {eff }}$ by the factor of $(1-d \omega)^{-1}$, because the presence of cracks simply reduce the intact area subjected to loading with a given total force. This results in the reduction

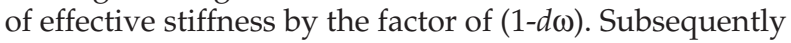

$$
d k / k=-\lambda l^{1-m} d l
$$

Obviously, the power law is only possible if $m=2$. Then the solution of the above differential equation reads:

$$
f(l)=\lambda l^{-2}, \quad \alpha=-\lambda, \quad k \sim l^{-\lambda}, \quad \tau_{\text {eff }} \sim l^{\lambda}
$$

The influence of the interaction on the average stress intensity factor scales inversely to the scaling of stiffness, therefore the criterion of crack grows reads

$$
K_{I I}=K_{I I c}, \quad K_{I I} \sim \frac{F}{\sqrt{\pi l}} l^{\lambda} \sim l^{\lambda-1 / 2}
$$

Therefore, the self-similarity will be preserved if

$$
\lambda=1 / 2
$$

The total dimensionless concentration of cracks with sizes in the range from $l_{0}$ to $l_{\max }$ is

$$
\Gamma_{0}=\frac{1}{2} \ln \frac{l_{\max }}{l_{0}}
$$

Thus, the self similar distribution of parallel sliding zones in an infinitesimally thin layer is stable with respect to their growth.

\section{SELF-SIMILAR CASCADES AND THE RESULTING MICROSEISMIC ENERGY DISTRIBUTION}

If we assume that the self-similarity of the size distribution of sliding zones is maintained, then the process of their evolution can be described as follows. The new sliding zones which are initially the zones of average length $l_{0}$ induced as a result of mining operations will violate the self-similarity. Therefore, the self-similarity will have to be restored by growing these new cracks (or some of them) to the highest lengths or by propagating them to some intermediate lengths 
followed by increasing the lengths of some other cracks. This cascade of propagating sliding zones will result in a new distribution with the same minimal length $l_{0}$ but with an increased maximal one, $l_{\max }$.

In order to model this cascade of propagating cracks consider a level $l$. Since the cracks grow in such a way that the concentration factor, $\lambda$, remains constant (see (29)), the number of crack grown to length $l$ should equal the number of cracks of length $l$ starting their growth in the process of rearrangement. Let $g(l)$ be a fraction of cracks grown to length l. Due to self-similarity this distribution should be expressed by a power law with a certain exponent $k$ :

$$
g(l)=\frac{\gamma}{l^{k}}
$$

Subsequently the distribution of cracks grown to length $l$

$$
f(l) g(l)=\frac{\lambda \gamma}{l^{2+k}}
$$

Consider now the energy distribution of microseismic events emitted by the cascade. Suppose the microseismic event is associated with a growth of a crack (sliding zone) from a certain length $l_{1}$ to length $l$. We will assume that this growth happens dynamically such that the energy emitted by the crack, $\mathrm{E}(l)$ is approximately equal to the change in elastic energy associated with the displacement of the crack faces. Since the crack growth is caused by the crack interaction, represented in this model by the uniform effective stress $\tau_{e f f}$ the energy in the considered $2 \mathrm{D}$ case is $\mathrm{E}(l) \sim \tau_{\text {eff }}\left(l^{2}-l_{1}^{2}\right)$. Assuming that the majority of growing cracks considerably increase their lengths, $l>>l_{1}$ and taking into account the last equation of (27) and equation (29) one obtains

$$
\mathrm{E}(l)=\kappa l^{5 / 2}
$$

One can now obtain the energy distribution expressed by the probability of events with energy greater that a certain level $U$ :

$$
P\{\mathrm{E}>U\}=\frac{1}{k+1}\left(\frac{\kappa}{U}\right)^{\frac{2}{5}(k+1)}
$$

This is a Guttenberg-Richter law expressed in terms of the energy rather than magnitude. After relating the exponent $2(k+1) / 5$ to the empirical one it is possible to find $k$.

\section{DETERMINATION OF THE MODEL PARAMETERS AND RISK ASSESSMENT}

In order to determine the parameters of the model we consider statistical moments of energy:

$$
\begin{aligned}
& \left\langle\mathrm{E}^{n}\right\rangle=\int_{l_{0}}^{l_{\max }} \mathrm{E}(l)^{n} f(l) g(l) d l \\
& =\frac{\lambda \gamma \kappa^{n}}{1+k-2.5 n}\left(\frac{1}{l_{0}^{1+k-2.5 n}}-\frac{1}{l_{\max }^{1+k-2.5 n}}\right)
\end{aligned}
$$

Thus by measuring the consecutive energy moments $<\mathrm{E}^{n}>$ one can determine remaining parameters, $\kappa, l_{0}, l_{\max }$ and the combination $\lambda \gamma$. Parameter $\lambda$ in this combination should be determined separately, for instance by considering progressive fault sliding due to advancing of an excavation, since this parameter together with $l_{0}$ and $l_{\max }$ determine effective stiffness of the fault. In this case the back analysis of the continuous displacement monitoring of the surface of the advancing excavation will be needed.
When these parameters are found the rock burst hazard assessment can be performed. Firstly, the maximum energy of a microseismic event can be determined:

$$
\mathrm{E}_{\max }=\kappa l_{\max }^{5 / 2}
$$

If this energy exceeds the safe level, $\mathrm{E}_{\text {safe }}$ then the microseismic event is considered hazardous. The risk of a hazardous microseismic event can be estimated as

$$
\begin{aligned}
& P_{\text {hazard }}=\int_{\left(\mathrm{E}_{\text {safe }} / \mathrm{\kappa}\right)^{2 / 5}}^{l_{\max }} g(l) d l \\
& =\left\{\begin{array}{l}
\frac{\gamma}{k-1}\left(\left(\frac{\kappa}{\mathrm{E}_{\text {safe }}}\right)^{\frac{2}{5}(k-1)}-\left(\frac{\kappa}{\mathrm{E}_{\max }}\right)^{\frac{2}{5}(k-1)}\right), \quad \text { if } k \neq 1 \\
\frac{2}{5} \ln \frac{\mathrm{E}_{\text {max }}}{\mathrm{E}_{\text {safe }}}, \quad \text { if } k=1
\end{array}\right.
\end{aligned}
$$

\section{CONCLUSIONS}

We have considered a specific mechanism of microseismicity associated with the propagation of sliding zones within a fault zone in such a way that the distribution of sizes of the sliding zones maintains self-similar. This is of course a very strong assumption. If the persistent observations of Guttenberg-Richter law, both at the scale of earthquakes and at the scale of mining-induced microseismicity, is a sufficient reason to believe that the self-similarity is the prevailing characteristics of the fault sliding, then we have a method of hazard assessment of rock bursts. It is essential that not all parameters needed for the hazard assessment could be determined from the microseismic measurements; these must be complemented by the observations of the process of the fault deformation. This can be done indirectly by observing the effect of the fault deformation on the approaching excavations by means of continuous displacement monitoring and back analysis. Specific methods for such monitoring still need to be developed.

\section{ACKNOWLEDGMENTS}

The author acknowledges the financial support through the Australian Research Council Large Grant (A00104937) and the financial support by the Australian Computational Earth Systems Simulator - Major National Research Facility (ACcESS MNRF).

\section{REFERENCES}

Bak, P. and Tang, C. (1989) Earthquakes as a self-organised critical phenomenon. J. Geophys. Res., 94B (11), pp. 15,635-15,637.

Barton, C.A. and Zoback, M.D. (1992) Self-similar distribution and properties of macroscopic fractures at depth in crystalline rock in the Cajon Pass scientific drill hole. J. Geophys. Res., 97B (4), pp. 5181-5200.

Chakrabati, B.K. and Benguigui, L.G. (1997) Statistical Physics of Fracture and Breakdown in Disordered Systems. Clarendon Press. Oxford.

Chopard, B. and Droz, M. (1998) Cellular Automata Modelling of Physical Systems. Cambridge University Press.

Dubois, J. (1998) Non-Linear Dynamics in Geophysics. John Wiley and Sons, Chichester, New York, Weinheim, Brisbane, Singapore, Toronto.

Dyskin, A.V. (2001) Self-similar crack distributions: Mechanics of formation and method of modelling. Intern. J. Fracture, 112, pp. L41-46.

Dyskin, A.V. (2002) Self-similar crack patterns induced by spatial stress fluctuations. Fatigue and Fracture of Engineering Materials and Structures, 25, pp. 187-200.

Dyskin, A.V. (2004) Effective characteristics and stress concentrations in materials with self-similar microstructure. Int. J. Solids Struct. (in print).

Dyskin, A.V., Galybin, A.N. and Brady, B.H. (1998) Catastrophic sliding over a fault caused by accumulation of dilation zones. Mechanics of Jointed and Faulted Rock (MJFR-3), H.P. Rossmanith (ed.), Balkema, pp. 69-74. 
Galybin, A.N. and Odintsev, V.N. (1993) The propagation of shear cracks near tabular stope. Assessment and Prevention of Failure Phenomena in: Rock Engineering, Pasamehmetogly et al. (eds). Balkema, Rotterdam, pp. 193-198.

Gibowicz, S.J. and Kijko, A. (1994) An introduction to Mining Seismology. San Diego, New York, Boston, London, Sydney, Tokyo, Toronto: Academic Press.

Gillespie, P.A., Howard, C.B., Walsh, J.J. and Watterson, J. (1993) Measurement and characterisation of spatial distribution of fractures. Tectonophysics, 226, pp. 113-141.

Krajcinovic, D. (1996) Damage Mechanics. Elsevier, Amsterdam, Lausanne, New York, Oxford, Shannon, Tokyo.

Mishnaevsky, L. (1998) Damage and Fracture in Heterogeneous Materials. Modelling and Application to the Improvement of Drilling Tool. Balkema/Rotterdam/Brookfield.

Nishiuma, S., Hasegawa, Y. and Miyazima, S. (1996) Percolated cracks in a solid containing many initial defects. Fractals, 4 (3), pp. 377-384.

Olding, N.E. (1992) Network properties of a two-dimensional nature fracture pattern. PAGEOPH, 138, pp. 95-114.

Patton, F.D. (1966) Multiple modes of shear failure in rock. Proceedings of the First Congress of ISRM. Lisbone, 1, pp. 509-513.

Redner, S. (1990) Fragmentation. In: Herrmann, H.J., Roux, S. (Eds.) Statistical Models for the Fracture of Disordered Media. North-Holland, Amsterdam, Oxford, New York, Tokyo, pp. 321-348.

Sadovskiy, M.A. (1983) Distribution of preferential sizes in solids. Transactions USSR Academy of Sciences. Earth Science Series, 269 (1), pp. 8-11.

Sahimi, M. and Goddard, J.D. (1986) Elastic percolation models for cohesive mechanical failure in heterogeneous systems. Physical Review B, 33 (11), pp. 7848-7851.

Salganik, R.L. (1973) Mechanics of bodies with many cracks. Mech. of Solids, 8, pp. $135-143$.

Scholz, C.H. (1990) The Mechanics of Earthquakes and Faulting. Cambridge University Press, Cambridge, New York, Port Chester, Melbourne, Sydney.

Scholz, C.H. and Aviles, C. (1986) The fractal geometry of faults and folding. In: Das, S., Boatwright, J., Scholz, C.H. (Eds) Earthquake Source Mechanics. Monograph series, 37. Washington, D.C.: American Geophysical Union, pp. 147-155.

Tada, H., Paris, P.C. and Irwin, G.R. (1985) The stress analysis of cracks. Handbook. Third edition. Vol. II. New York: ASME Press.

Turcotte, D.L. (1993) Fractals and Chaos in Geology and Geophysics. Cambridge University Press.

Vavakin, A.S. and Salganik, R.L. (1978) Effective elastic characteristics of bodies with isolated cracks, cavities, and rigid nonhomogeneities. Mech. of Solids, 13 (2), pp. 87-97.

Yamamoto, H., Kojima, K. and Tosaka, H. (1993) Fractal clustering of rock fractures and its modelling using cascade process. In: Pinto da Cunha (Ed.), Scale Effects in Rockmasses 93. Balkema, Rotterdam, pp. 81-86. 